Kreis D. J., Plasencia G., Augenstein D., Davis J. H. H. Y., Echenique M., Vopal J., Byers B. \& Gomez G. (1986) Preventable trauma deaths: Dade County, Florida. Journal of Trauma 26, 649-653.

Williams L., Muwanga C. L., Worlock P. H., Moran C. G., \& Price K. A. (1991) Teaching trauma management in the accident and emergency department. Archives of Emergency Medicine 8, 205-209.

\title{
Pulsed electromagnetic energy and pre-tibial lacerations
}

Sir

I wish to comment on the letter from Muirhead et al. (Archives of Emergency Medicine 8(2) 152-154). Their study appears to have been well planned but the conduct of it must have met unanticipated problems. They state that, after exclusions, 48 patients were entered into both the treatment and the control groups but Table 3 which reports healing times only includes 15 treated patients and 13 controls. It is not clear from the text whether Table 3 relates only to female patients under 60 years of age but it does show a clear preference for the patients with pulsed electromagnetic energy.

It is unfortunate that the authors chose to do this study using a Curapuls machine which is a maverick device among machines of this nature. The original Diapulse machine and the widely used Megapulse machine use a pulse width of $50-65 \mu \mathrm{s}$, Curapuls uses $400 \mu \mathrm{s}$. The underlying reason behind using a pulsed field is that the biological action results from the on/off effect and any heat generated by the field will be dissipated during the resting phase and before the next pulse occurs. In my own studies using the Diapulse device I have found that 400 pulses $\mathrm{s}^{-1}$ with a $65 \mu$ s pulse width applied for $20 \mathrm{~min}$ gives a definite beneficial therapeutic effect (Wilson, 1972).

D. H. WILSON

Dean of Postgraduate Medical Education

The University of Leeds

Leeds

\section{REFERENCES}

Wilson D. H. 1972. British Medical Journal 2, 269-270.

\section{Massive haemothorax from central venous catheterization: a note of caution}

Sir

The various techniques for central venous catheterization are an essential component of the armamentarium of physicians providing emergency care (Parsa \& Tabora, 1986; Putterman, 1986). Though the list of complications associated with this procedure is quite long, proper attention to technique and to patient management can reduce attendant morbidity and mortality to an acceptable minimum (McGoon et al., 1979).

Perforation of venous vascular structures is a relatively rare, but potentially lethal, complication of central venous catheterization. It is recommended that a 
post-insertion chest film should always be carried out to check for, and diagnose, possible complications. Nevertheless, some clinicians tend to postpone radiographic control, if clinical criteria indicate that the catheter is properly positioned and is functioning normally (Conces \& Wolden, 1984; Gamulin et al., 1986). We describe a patient with massive haemothorax from a central venous catheter, for whom an appropriately timed chest film might have prevented the resultant morbidity.

\section{CASE REPORT}

A 90-year-old woman with known dementia was admitted to the Ezrath Nashim Hospital because of mental deterioration. During the in-hospital stay the patient progressively became oliguric and then anuric. To monitor hydration adequately, a 16-gauge vialon single lumen catheter through-the-needle assembly (Intracath, Deseret Medical) was inserted by the infraclavicular approach to the right subclavian vein. Blood returned freely from the catheter, after insertion into the superior vena cava. A chest film could not be obtained at that time, for technical reasons. Central venous pressure was measured at $9 \mathrm{~cm} \mathrm{H}_{2} \mathrm{O}$, and fluid infusion was begun. Three $\mathrm{h}$ later the patient became severely dyspneic. Physical examination revealed a respiratory rate of $24 \mathrm{~min}^{-1}$, with dullness to percussion and decreased breathing sounds over the lower half of the right hemithorax. An emergency chest film was obtained, and demonstrated a diffuse ground glass appearance of the right hemithorax. Due to the massive pleural fluid accumulation, the catheter tip could not be accurately visualized. Catheter inspection again revealed free blood return. Thoracocentesis yielded $900 \mathrm{ml}$ of bloody fluid, and led to a dramatic improvement in the patient's respiratory status. A repeat blood count revealed a drop in the haematocrit, signifying significant blood loss into the pleural space. The central venous catheter was completely withdrawn, and no further accumulation of fluid or blood was noted.

\section{DISCUSSION}

Perforation of the superior vena cava by a central venous catheter proximal to the junction of the superior vena cava and right atrium may result in hydrothorax and hydromediastinum (Apps, 1977). Perforation beneath this point leads to cardiac tamponade (Jay \& Kehler, 1987). When vascular erosion occurs, the catheter tip does not usually cause a large perforation in the vascular wall. In fact, the exact site at times cannot even be identified at autopsy (Chabanier $e t$ al., 1988). Therefore, haemorrhage is generally slight, and the fluid found on thoracocentesis is bloody only rarely.

Massive haemothorax from iatrogenic vascular perforation is reported only rarely (Shiloni et al., 1985). Possibly, one can attribute the haemothorax in our patient to not advancing the large bore catheter over a guidewire (by Seldinger technique) 
which perhaps led to a larger initial intimal injury. Secondly, although it was possible to aspirate blood from the catheter, which indicated proper vascular positioning, the tip obviously was outside the vein and communicated with the pleural space. This phenomena has been described previously, though perforation is usually suspected when infusion rates are disturbed, or when blood return is diminished through the catheter (Ellis et al., 1988). In these uncommon cases such as ours, where it is possible to aspirate blood from the catheter even though the tip has perforated the vessel, the explanation is that the perforating tip indeed lies outside the vessel lumen, in a small collection of blood caused by the injury (McMahon, 1973). Another factor contributing to the perforation of the venous wall in this case was the relative stiffness of the catheter inserted. It has been demonstrated that the risk of perforation significantly decreases by selection of catheters with soft and flexible tips (Bersten et al., 1988).

Central venous catheterization is a procedure with great potential benefit to the patient, and accordingly is widely used for many diagnostic and therapeutic applications. However, the clinician should be aware constantly of the possibility of the patient developing a iatrogenic complication, related to the insertion, advancement and maintenance of the catheter. Technically undemanding and uncomplicated insertion, satisfactory infusion rates and adequate return of blood on aspiration do not guarantee correct positioning of the catheter, nor do they rule out occurence of complications such as vascular erosions and resultant hydroand haemothorax. We wish to re-emphasize the absolute neccessity of a control chest film immediately after central venous catheter insertion, and at appropriate intervals thereafter. The technical inability to perform such a radiograph should be considered close to an absolute contraindication for elective percutaneous central venous catheterization.

\section{PUTTERMAN}

Department of Internal Medicine

Hadassah University Hospital

Kiryat Hadassah

Jerusalem

\section{REFERENCES}

Apps M. C. P., Clark J. M. F. \& Skeater S. J. (1977) Hydrothorax, a complication of the insertion of central venous cannulae. Intensive Care Medicine 3, 41-43.

Bersten A. D., Williams D. R. G. \& Phillips G. D.(1988) Central venous catheter stiffness and its relation to vascular perforation. Anaesthesia Intensive Care 16, 342-357.

Chabanier A., Dany F., Brutus P. \& Vergnoux H. (1988) Iatrogenic cardiac tamponade after central venous catheterization. Clinical Cardiology 11, 91-99.

Conces D. J. \& Wolden R. W. (1984) Aberrant locations and complications in intial placement of subclavian vein catheters. Archives of Surgery 119, 293-295.

Ellis L. M., Vogel S. B. \& Copeland E. M. (1989) Central venous catheter erosions: Diagnosis and clinical management. Annals of Surgery 209, 475-478.

Gamulin Z., Bruckner J. C., Forster A., Simonet F. \& Rouge J. C. (1986) Multiple complications after internal jugular vein catheterization. Anaesthesia 41, 408-412.

Jay A. W. L. \& Kehler C. H. (1987) Heart perforation by central venous catheters. Canadian Journal of Anaesthesia 34, 333-335. 
McGoon M. D., Benedetto P. W. \& Greene B. M. (1979) Complications of percutaneous central venous catheterization: A report of two cases and review of the literature. John Hopkins Medical Journal 145, 1-6.

McMahon M. J. (1973) Hazards of central venous catheterization (letter). British Medical Journal 3, 353.

Parsa M. H. \& Tabora F. (1986) Central venous access in critically ill patients in the emergency department. Emergency Medicine Clinics of North America 4, 709-43.

Putterman C. (1986) Central venous catheterization: Indications, techniques, complications, management. Acute Care 12, 219-43.

Shiloni E., Meretyk S. \& Weiss J. (1985) Tension haemothorax: An unusual complication of central venous catheterization. Injury 16, 385-86.

\section{Teaching of basic life support}

\section{Sir}

Current teaching of basic life support indicates that, prior to commencing any cardiopulmonary resuscitation, adequate assessment of the situation and of the patient is mandatory. Two recent events have highlighted the importance of this teaching to us and we feel that the lessons are worth drawing to a wider audience.

We have just completed an assessment of the ability of 106 medical students to perform basic life support adequately. The assessment comprised an MSQ to assess theoretical knowledge and a practical test on a mannequin. Only one student was unable to perform the actual mechanics of cardiopulmonary resuscitation upon the mannequin. However, in total, just under one third of the students failed the overall exam. This was almost exclusively on account of a deficiency in either theoretical knowledge of the initial assessment of the patient or inability to initially assess the mannequin in the practical part o the examination.

Shortly after this assessment was completed a middle-aged male was brought to the A\&E Department having undergone treatment by bystanders. The bystander assessment indicated that the man was breathing and had a pulse. He was consequently transported to Accident and Emergency in the recovery position. Continuous assessment was made throughout the journey and we were assured that all vital signs were present on arrival. Assessment in the Accident and Emergency Department revealed asystole, a mouth and pharynx blocked with vomitus, fixed dilated pupils and marked anterior lividity. The man was pronounced dead soon after his arrival to A\&E.

If bystander cardiopulmonary resuscitation is to be effective the patient must be assessed accurately. Our experience is that a student acquires the mechanical skills of cardiopulmonary resuscitation with ease. However, it would appear that initial assessment of a collapsed patient which would determine whether or not resuscitative measures are instituted, may present a quandary to the rescuer. We believe that anyone embarking on basic life support teaching, to whatever group, must stress the importance of adequate assessment of the patient. Only then will rescuers confidently embark on resuscitative measures thus avoiding the tragedy of withholding such life saving measures from those patients who most need them.

\section{A. RODGER, J. FERGUSON \& T. F. BEATTIE} Accident and Emergency Department

Aberdeen Royal Infirmary

Aberdeen 